\title{
Avaliação do Tratamento Preventivo e Manejo Terapêutico da Osteorradionecrose dos Maxilares em Pacientes Submetidos a Radioterapia: Uma Revisão Integrativa.
}

\section{Evaluation of Preventive Treatment and Therapeutic Management of Maxilary Osteorradionecrosis in Patients Undergoing Radiotherapy: Integrative Review}

Amanda Thalya Soares da Silva ${ }^{1}$, Ana Beatriz Lima de Oliveira ${ }^{1}$, Guilherme Rodrigues Wanderley de Oliveira ${ }^{1}$, Hellen Kevillyn Brito de Souza ${ }^{1}$, Marianne Pereira de Moura ${ }^{1}$, Mariana

Alves Lemos ${ }^{2}$, Rebeca de Almeida Buriti da Silva ${ }^{1}$, Héberte de Santana Arruda ${ }^{3}$

\begin{abstract}
RESUMO
A osteorradionecrose é complicação em decorrência da radioterapia, na qual varia de acordo com a extensão e gravidade da sintomatologia. Sendo a atenção odontológica indispensável para reduzir o desenvolvimento e progressão da doença. Objetivo: Revisar na literatura os tratamentos preventivos e terapêuticos da Osteorradionecrose. Metodologia: Trata-se de uma revisão integrativa de literatura de artigos em inglês, publicados nos últimos 10 anos, e indexados nas Bases de Dados: Biblioteca Virtual em Saúde e Pubmed/Medline. Com os descritores "radiotherapy" "osteoradionecrosis", "head and neck neoplasms", combinados através do operador booleano "AND'. Por fim, quatro artigos compuseram a amostra do estudo. Resultados: O uso de L-PRF em pacientes com câncer após radioterapia não parece oferece nenhum benefício adicional aos protocolos utilizados. Ademais, o tratamento à longo prazo com PENTOCLO é eficaz, e curativo para a osteorradionecrose (ORN) pois induz a cicatrização óssea. Conclusão: Faz-se necessário a realização de mais pesquisas acerca da temática, a fim de auxiliar o conhecimento dos profissionais de saúde e promover avanços no tratamento e manejo desses pacientes.
\end{abstract}

Palavras-chave: Radioterapia; Osteorradionecrose; Cancer de cabeça e pescoço.

\begin{abstract}
Osteoradionecrosis is a complication resulting from radiotherapy, which varies according to the extent and severity of the symptoms. Dental care is essential to reduce the development and progression of the disease. Objective: To review the preventive and therapeutic treatments for osteoradionecrosis in the literature. Methodology: This is an integrative literature review of articles in English, published in the last 10 years, and indexed in the Databases: Virtual Health Library and Pubmed / Medline. With the descriptors " radiotherapy " "osteoradionecrosis',', 'head and neck neoplasms', combined using the Boolean operator "AND'. Finally, four articles comprised the study sample. Results: The use of L-PRF in cancer patients after radiotherapy does not seem to offer any additional benefit to the protocols used. Furthermore, long-term treatment with PENTOCHL is effective, and curative for osteoradionecrosis $(\mathrm{ORN})$ as it induces bone healing. Conclusion: It is necessary to carry out more research on the subject, an end to knowledge of health professionals and promote advances in the treatment and management of these patients.
\end{abstract}

Keywords: Radiotherapy; Osteoradionecrosis; Head and Neck Neoplasms. 


\section{INTRODUÇÃO}

O câncer é um problema de saúde global significativo, que afeta milhões de pessoas no mundo (BENNETT et al., 2017). Dentre os tipos de cânceres, cumpre destacar o câncer de cabeça e pescoço (CCP), correspondente aos tumores na região de cavidade oral, faringe e laringe. Nesse sentido, dentre os tipos de terapêutica, a radioterapia é o tratamento preconizado, visto que, surge como uma forma efetiva ao tratamento de malignidades da região de cabeça e pescoço, podendo ser usada para lesões inoperáveis, adjuvante ao tratamento cirúrgico e juntamente com o tratamento quimioterápico (SILVA, 2017). Entretanto, embora aumente significativamente a sobrevida dos pacientes, possui dentre os efeitos colaterais o desenvolvimento de reações indesejáveis, tais como, a xerostomia, mucosite, lesões vasculares e osteorradionecrose (ROBARD et al., 2014; BENNETT et al., 2017; SILVA, 2017).

Partindo desse pressuposto, a ORN caracteriza-se pela presença de áreas com ulceração ou necrose, presentes no tecido mole, associada a uma área de exposição de osso necrótico, no qual não cicatriza naturalmente. Assim, dentre suas principais manifestações clínicas, destaca-se as infecções secundárias, dor, parestesia, fístulas, e dificuldade na fala. Dessa forma, a ORN está associada a considerável morbidade e perda na qualidade de vida nos pacientes com câncer (EL-RABBANY et al., 2019). Torna-se evidente a necessidade de uma avaliação odontológica dos pacientes antes do tratamento radioterápico, e posteriormente a assistência ao tratamento restaurador, endodôntico e cirúrgico, visto que, o acompanhamento odontológico pré, trans e pós radioterápico promove melhor qualidade de vida aos pacientes (SILVA, 2017).

Embora as repercussões da doença sejam amplamente descritas na literatura, esta condição ainda apresenta etiologia não totalmente elucidada, assim como um tratamento complexo e demorado (EL-RABBANY et al., 2019; KOLOKYTHAS et al., 2018). O Instituto Nacional do Câncer ressalta a importância do manejo terapêutico e preventivo da doença, seja pela pré-irradiação oral, triagem dentária, bem como, prevenção pós-irradiação subsequentes, para dessa maneira, reduzir a necessidade de tratamento dentário invasivo (EL-RABBANY et al., 2019). Desta forma, o presente trabalho visa realizar uma revisão integrativa da literatura, com o intuito de analisar os tratamentos preventivos e terapêuticos da Osteorradionecrose dos maxilares. 


\section{METODOLOGIA}

O presente estudo trata-se de uma revisão integrativa, devendo seguir de maneira criteriosa o procedimento preconizado, pretendendo identificar e selecionar as principais características das publicações. A revisão foi desenvolvida seguindo as bases da metodologia Prisma - Preferindo reportar os Ensaios Clínicos Randomizados-Essa modalidade de estudo é norteada através de seis fases distintas: Escolha do tema e elaboração da pergunta de pesquisa para a revisão; Determinação da estratégia de busca por meio de descritores e operadores booleanos; Seleção dos estudos por meio da pesquisa avança, considerando os critérios de inclusão; Análise crítica; Avaliação e categorização do conteúdo; Tabulação dos resultados.

A pergunta norteadora do proposto estudo foi: Quais procedimentos preventivos e terapêuticos para a Osteorradionecrose? O recorte temporal consistiu em revisar as publicações dos últimos 10 anos, Janeiro/2011 a Janeiro/2021. Os critérios de inclusão definidos para selecionar os estudos foram: estudos publicados em português ou inglês; publicações disponíveis em formato de artigos completos (originais), no período de janeiro/2011 a janeiro/2021. Foram excluídas teses, dissertações, monografias, cartas ao editor, índices incomuns, revisões e artigos que embora apresentassem os descritores selecionados, não abordaram diretamente a temática.

Esta revisão foi produzida através de seis passos: $1^{\text {a }}$ Fase: elaboração da pergunta norteadora; $2^{\mathrm{a}}$ Fase: busca ou amostragem na literatura; $3^{\mathrm{a}}$ Fase: coleta de dados; $4^{\mathrm{a}}$ Fase: análise crítica dos estudos incluídos; $5^{\mathrm{a}}$ Fase: discussão dos resultados;

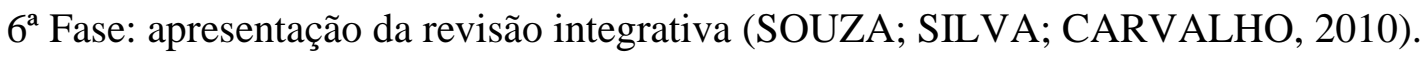

A revisão foi realizada com base na metodologia Prisma - Preferred Reporting Items for Systematic Reviews and MetaAnalyses (MOHER et al., 2009). Para avaliação do nível de evidência dos estudos incluídos na revisão foi utilizado a pirâmide de níveis de evidência. Os estudos para tratamento e prevenção são hierarquizados de acordo com o grau de confiabilidade que está relacionado à qualidade metodológica dos mesmos (COOK et al., 1995). Todos os artigos selecionados têm como nível de evidência 1B, consequentemente, o grau de recomendação dessa Revisão Integrativa é A, segundo o Nível de Evidência Científica por tipo de Estudo, do Centro de Oxford (2009). 
Para identificar as publicações que compuseram a amostra deste estudo, realizou-se uma busca online, mediante levantamento na Biblioteca Virtual em Saúde (BVS), e no PubMed/Medline, que foi utilizada porque além de apresentar um grande quantitativo da produção científica relevante na área da saúde, permite obter artigos originais de alto impacto. Referências duplicadas foram excluídas. As listas foram comparadas e, no caso de divergências, as decisões foram tomadas com o auxílio de um terceiro autor, com experiência no tema investigado, e após discussão com base nos critérios determinados de inclusão e exclusão, como forma de garantir rigor ao processo de seleção dos artigos. Foram utilizados os seguintes descritores padronizados e disponíveis em Medical SubjectHeadings: 'radiotherapy'” AND (osteoradionecrosis", "head and neck neoplasms" os quais foram combinados através do operador booleano “AND” de forma a restringir a amplitude da pesquisa e os termos.

\section{RESULTADOS}

Durante o processo de busca, a pesquisa bibliográfica mostrou 1.162 artigos. Destes, 1.128 na Medline/PubMed e 34 na Lilacs/BVS. Após a realização da primeira triagem, aplicando os critérios exclusão restaram 24 artigos. Após a leitura dos títulos e resumos, 07 artigos foram selecionados. Ao analisar esses remanescentes, 03 foram excluídos por não atenderem os objetivos da pesquisa. Os estudos que atenderam aos critérios de busca previamente estabelecidos foram selecionados para leitura integral. Dessa maneira, 04 artigos compuseram a amostra final, restando assim, apenas ensaios clínicos randomizados, e assim, artigos que cumpriram todos os critérios de inclusão e exclusão desta revisão. Casos de discordância entre os autores foram resolvidos após a discussão. Planilhas de coleta de dados sendo previamente definidas foram empregadas para cada publicação selecionada.

A tabela 1 apresenta a distribuição dos estudos, cruzando os autores, título, país, população e amostra, seguindo os descritores utilizados nas bases de dados. Assim, as publicações foram classificadas em (A1, A2, A3 e A4), para dar característica a cada estudo selecionado na revisão. Os estudos foram publicados no período de 2011 a 2020, seguindo os critérios dos últimos dez anos. Ademais, os países de publicação foram: 
Estados Unidos, Espanha e Suíça, não havendo assim, nenhuma publicação brasileira. Seguindo os critérios de busca, foram utilizados estudos no idioma português e inglês. O delineamento do estudo resulta em 4 estudos, sendo (A1, A2 e A4) estudo de Ensaio Clínico Randomizado, e (A3) Ensaio Clínico Randomizado e Estudo Duplo-cego Controlado. Os objetivos do estudo são claros e de fácil compreensão.

Figura 1. Fluxograma de elegibilidade e inclusão dos artigos científicos.

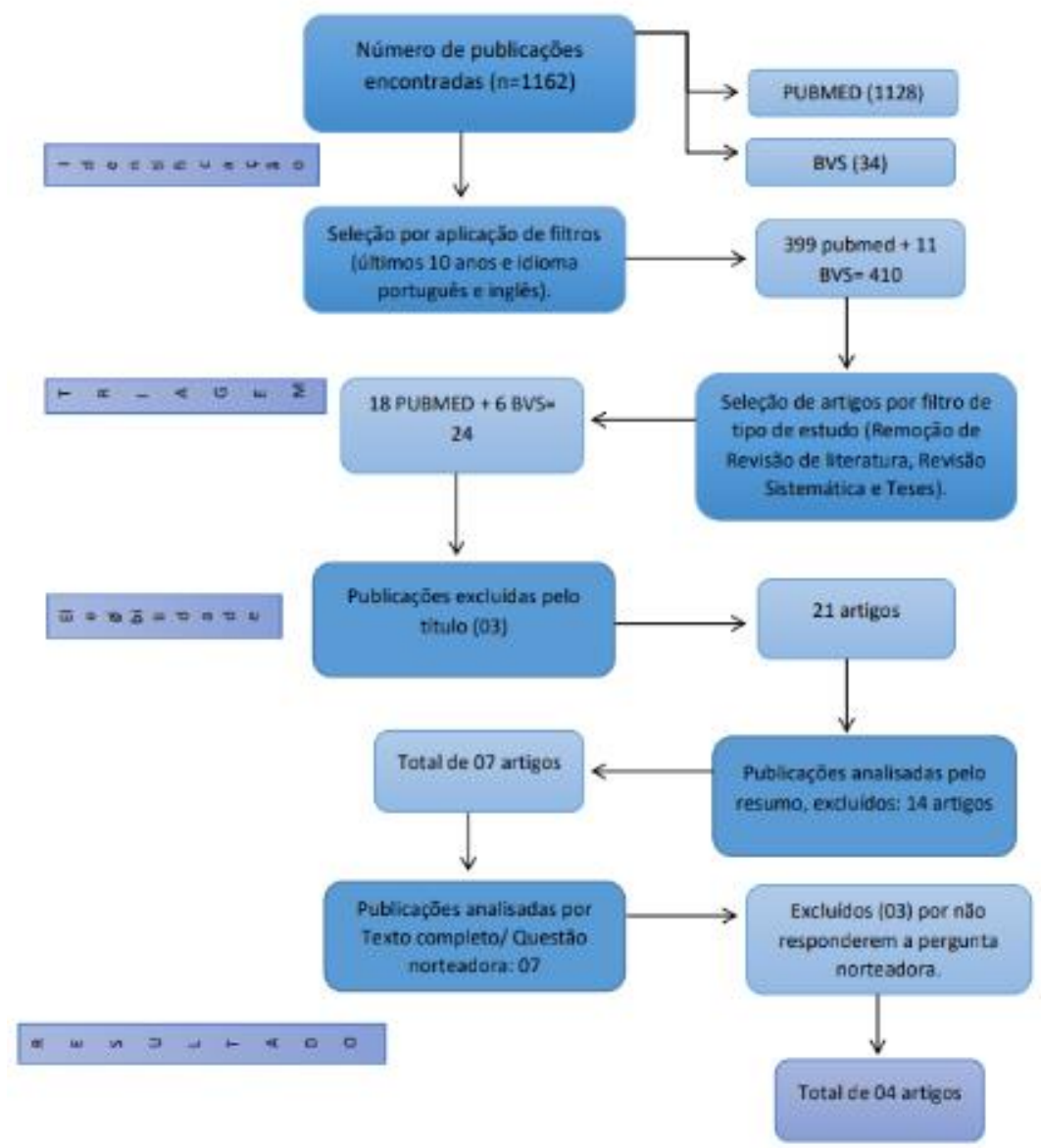


Tabela 1. Distribuição dos estudos segundo código, autoria e ano título, país, amostra e tipo de Estudo:

\begin{tabular}{|c|c|c|c|c|c|c|}
\hline $\begin{array}{c}\text { Código } \\
\text { do } \\
\text { Artigo }\end{array}$ & $\begin{array}{l}\text { Autore } \\
\text { s/Ano }\end{array}$ & Título & País & $\begin{array}{c}\text { Amostra } \\
\text { (n) }\end{array}$ & $\begin{array}{l}\text { Tipo de } \\
\text { Estudo }\end{array}$ & Objetivo \\
\hline A1 & $\begin{array}{l}\text { PALMA, } \\
\text { L.F., et } \\
\mathrm{al} / 2020 \text {. }\end{array}$ & $\begin{array}{l}\text { Leukocyte- and } \\
\text { platelet-rich fibrin does } \\
\text { not provide any } \\
\text { additional benefit } \\
\text { for tooth extraction in } \\
\text { head and neck cancer } \\
\text { patients } \\
\text { radiotherapy: } \\
\text { a randomized clinical } \\
\text { trial }\end{array}$ & Espanha & $\begin{array}{c}23 \\
\text { pacientes }\end{array}$ & $\begin{array}{l}\text { Ensaio Clínico } \\
\text { Randomizado }\end{array}$ & $\begin{array}{l}\text { Avaliar a eficácia da } \\
\text { fibrina rica em leucócitos } \\
\text { e plaquetas (L-PRF) na } \\
\text { prevenção de ORN após } \\
\text { extração dentária em } \\
\text { pacientes com CCP após- } \\
\text { irradiado, bem como } \\
\text { outras complicações pós- } \\
\text { operatórias }\end{array}$ \\
\hline A2 & $\begin{array}{l}\text { DELANI } \\
\text { AN, S., } \\
\text { et } \\
\text { al/2011 }\end{array}$ & $\begin{array}{l}\text { COMPLETE } \\
\text { RESTORATION OF } \\
\text { REFRACTORY } \\
\text { MANDIBULAR } \\
\text { OSTEORADIONECR } \\
\text { OSIS } \\
\text { PROLONGED } \\
\text { TREATMENT WITH } \\
\text { A } \\
\text { PENTOXIFYLLINE- } \\
\text { TOCOPHEROL- } \\
\text { CLODRONATE } \\
\text { COMBINATION } \\
\text { (PENTOCLO): } \\
\text { A PHASE II TRIAL }\end{array}$ & $\begin{array}{l}\text { Estados } \\
\text { Unidos }\end{array}$ & $\begin{array}{c}54 \\
\text { pacientes } \\
\text { elegíveis }\end{array}$ & $\begin{array}{l}\text { Ensaio Clínico } \\
\text { Randomizado }\end{array}$ & $\begin{array}{l}\text { Avaliar se o Pentoclo é } \\
\text { eficaz em } \\
\text { osteorradionecrose de } \\
\text { mau prognóstico }\end{array}$ \\
\hline A3 & $\begin{array}{l}\text { BATST } \\
\text { ONE, M. } \\
\text { D., et } \\
\text { al./2012 }\end{array}$ & $\begin{array}{l}\text { Platelet rich plasma for } \\
\text { the } \\
\text { Prevention of } \\
\text { Osteoradionecrosis. A } \\
\text { double } \\
\text { Blinded randomized } \\
\text { cross over } \\
\text { Controlled trial. }\end{array}$ & $\begin{array}{l}\text { Estados } \\
\text { Unidos }\end{array}$ & $\begin{array}{c}22 \\
\text { pacientes }\end{array}$ & $\begin{array}{c}\text { Estudo } \\
\text { randomizado } \\
\text { duplo-cego } \\
\text { controlado }\end{array}$ & $\begin{array}{l}\text { Determinar se Plasma } \\
\text { rico em plaquetas (PRP) } \\
\text { em extrato dentário } \\
\text { poderiam ajudar a } \\
\text { prevenir } \\
\text { osteorradionecrose } \\
\text { (ORN) da mandíbula e } \\
\text { determinar seus efeitos na }\end{array}$ \\
\hline
\end{tabular}




\section{A4}

\begin{tabular}{l|l|}
$\begin{array}{l}\text { STUDE } \\
\text { R, G., et }\end{array}$ & Risk-adapted dental \\
al/2011 & modulated \\
& radiotherapy (IMRT).
\end{tabular}

Suíça

Ensaio Clínico

Randomizado

dor da ferida.

Avaliar a eficácia do

atendimento

odontológico adaptado ao

risco conforme refletido

pela taxa de

Osteorradionecrose de

nossos pacientes com

radioterapia modulada

por intensidade (IMRT).

Tabela 2. Distribuição dos estudos conforme métodos, resultados e conclusão

\section{Código}

do

Artigo

A1

\section{Método}

Foi realizado no Centro de Cirurgia

Bucomaxilofacial no Hospital Heliópolis e aprovado pelos comitês de ética competentes. Foram incluídos 23 pacientes que haviam sido submetidos a uma única extração dentária, com mais de 18 anos e apresentando uma história de convivência com radiografia conformacional para câncer de cabeça e pescoço. Os pacientes foram aleatoriamente designados para receber cirurgia e protocolos de drogas com coágulos de L-PRF para preencher e cobrir os soquetes de extração (Grupo de Teste=11 pacientes) ou sem ele (Grupo controle $=13$ pacientes). A randomização foi realizada por meio de uma tabela gerada por computador e o código de tratamento para cada paciente foi alocado em um número. Para o diagnóstico de Osteorradionecrose, os pacientes foram avaliados clinicamente por até 180 dias. Outras complicações pós-

\section{Resultados}

Dos 23 pacientes envolvidos no estudo, a idade média do Grupo Controle foi 65,5 anos e no Grupo de teste foi 59,2 em significância estatística. Diferença de escala (teste $t$ de Student; $\mathrm{P}=0,122)$. Da mesma forma, não houve diferença (teste do quiquadrado) entre os Grupos sobre sexo, cor da pele, uso de tabaco, álcool uso, localização do tumor primário, tipo histológico de tumor,

dose total de radiação, período pós-RT, quimioterapia e Cirurgia oncológica. No que diz respeito aos procedimentos cirúrgicos, foram 8 dentes
A falta de qualquer diferença significativa estatisticamente entre os dois grupos em relação às variáveis dos pacientes indica uma adequada homogeneidade da amostra. Em síntese, o uso de L-PRF em exodontias para pacientes com câncer cabeça e pescoço após radioterapia não parece oferecer nenhum benefício adicional aos protocolos cirúrgicos e medicamentosos propostos. 
operatórias (edema, alveolite, deiscência de sutura, sangramento contínuo e comunicação oroantral) também foram avaliadas neste período.

54 pacientes elegíveis previamente irradiados para câncer de cabeça e pescoço (entre 72 tratados) em média 5 anos. Recebeu ORN mandibular exteriorizado refratário por 1,4 $\pm 1,8$ anos, principalmente após cirurgia local e o oxigênio hiperbárico foi ineficaz. O comprimento médio do osso exposto (D) foi de $17 \pm 8 \mathrm{~mm}$ como ponto final primário, e a pontuação média subjetiva, objetiva, de manejo e avaliação analítica da lesão (SOMA) foi de $16 \pm 4$. Entre agosto de 2000 e agosto de 2008, todos os pacientes receberam PENTÓLOGO oral diário: 800 mg de pentoxifilina, 1.000 UI de vitamina E e $1.600 \mathrm{mg}$ de clodronato 5 dias por semana, alternando com $20 \mathrm{mg}$ de prednisona e $1.000 \mathrm{mg}$ ciprofloxacina 2 dias por semana. A duração do tratamento foi relacionada à cura consolidada.

Os pacientes foram recrutados no Hospitl clínica de cabeça e pescoço em 2003, durante um período de 12 meses. No momento do recrutamento, foram feitas superiores, 4 inferiores foram tratadas no grupo controle e 4 superiores e 7 inferiores no Grupo de Teste. Apenas um paciente do Grupo teste adquiriu dor pós-operatória no $3^{\circ}$ dia, reportando uma pontuação de 2. Não houve nenhuma diferença significativa entre os grupos. Não houve nenhum relato de desenvolvimento de Osteorradionecrose e complicação pós-operatório

O tratamento prolongado (16 \pm 9 meses) foi seguro e bem tolerado. Todos os pacientes melhoraram, com um exponencial Tial progressiva e redução significativa ( $p$ $<0,0001)$ do osso exposto (D), combinado com sequelas espontâneas em 36 pacientes. Todos os pacientes tiveram recuperação completa em uma média de 9 meses. Melhoria clínica foi medida em termos de descontinuação de analgésicos, nova fratura, fístula cutânea fechada e atraso melhora radiológica.

Durante o período do estudo, 22 pacientes se conheceram os critérios do estudo e consentiu em participar
O tratamento de longo prazo com PENTOCLO é eficaz, seguro e curativo para ORN refratário e induz cicatrização óssea com melhora significativa dos sintomas. Essas descobertas precisarão ser confirmadas em um teste julgamento dominado.

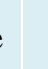


extrações dentárias na região posterior da mandíbula, no campo da radioterapia. Todos os pacientes selecionados possuíam um diagnóstico de câncer de cabeça e pescoço, sendo necessário um tratamento de radioterapia. Os pacientes eram excluídos do estudo se tivessem dentes abertos de forma patológica ou possuíssem infecção odontogênica ou periapical. Após isso, os pacientes randomizados no momento da cirurgia para receber o plasma rico em plaquetas (PRP). O grupo controle recebeu PRP colhido usando 3 PCCS (sistema de acordo com o fabricante), que foram colocadas nos soquetes com espuma de celulose e ativada com o sangue do paciente e cloreto de cálcio. Os pacientes foram revisados em 2 semanas, depois 2, 6, 9 e 12 meses. 5 anos após houve a revisão de todas as anotações

Os dados foram coletados em exposição óssea e dor (auto relatado através de uma escala Likert de 1 a 10 ). O teste exato de Fisher foi usado para determinar meu se houve alguma diferença entre o lado do tratamento e o pré-sentido de ORN. Para determinar se havia alguma diferença na dor pontuações dos lados do tratamento, um par de teste $\mathrm{t}$ de amostras foi usado para avaliar diferenças ao longo dos cinco pontos no tempo. Testes $\mathrm{t}$ de amostra pendentes foram usados para avaliar diferenças no tempo de extração dentária e dose a radiação para ORN e sem OR capital. Havia 5 mulheres (23\%) e 17 homens (77\%). Sua idade, demográfica detalhes e características de radioterapia. Local do tumor era desconhecido em cinco pacientes, era oro- Faríngea em nove pacientes, nasofarina- Geral em três pacientes, e a laringe hipofaringe em cinco pacientes. Três pacientes desenvolveram ORN durante o período de acompanhamento de 5 anos (um paciente teve ORN bilateral) dando uma taxa geral

de $14 \%$. ORN desenvolvido em três lados tratado com PRP e um lado controle. Esta diferença não foi estatisticamente significante significativo (valor $\mathrm{P}=0,61$, teste exato de Fisher).

Os escores médios de dor em cada um dos cinco os períodos de tempo são mostrados na Tabela 2. As avaliações são identificadas por um tamanho de amostra (n) inferior a 22 pacientes. Daí alguns cálculos são baseados em tamanhos de amostra menor que 22 pacientes. Em todo o seguinte períodos baixos, não houve

Diferenças nas pontuações de dor registradas entre $\mathrm{o}$ dor ou Cicatrização da mucosa em lados tratados com plasma rico em plaquetas. O plasma rico em plaquetas não mostra um benefício na prevenção da osteorradionecrose. A taxa de Osteorradionecrose é alta em comparação com outras séries publicadas e a profilaxia a remoção dos dentes molares deve ser questionada como medida preventiva.

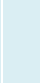

(n)


controle ou os lados do PRP.

A diferença nas pontuações

de dor entre o lado do

tratamento foi ligeiramente

diferente - em seguida, após

2 semanas (valor $\mathrm{P}=0,09$,

pareado- teste $\mathrm{t}$ de amostras).

Não houve associação entre o

desenvolvimento de ORN e o

site do

Tumor. Nem houve qualquer

associação entre a dose de

radioterapia ou tempo da

extração dentária à

radioterapia em $\mathrm{O}$

desenvolvimento de ORN.

A4 A incidência de Osteorradionecrose (ORN) na coorte de radioterapia modulada por intensidade (IMRT) foi comparado com a incidência após tratamento odontológico convencional ou mais invasivo. Esperandose encontrar um nível baixo e inalterado de incidência de ORN, comparando grupos $1 \mathrm{e}$ 2. Uma vez que a ORN ocorre em áreas expostas a altas doses. Foi realizado, também, um formulário do paciente usado para marcar áreas de risco para o atendimento odontológico adaptado ao risco.
A taxa de Osteorradionecrose de grau 2 foi semelhante nos grupos tratados convencionalmente e RaDC $(2 \%$ e $1 \%$, resp.); Osteorradionecrose de grau 3 não ocorreu pelo momento em que a análise foi conduzida. Como esperado, menos extrações foram realizadas na coorte $\mathrm{RaDC}$ (não extrações em $47 \%$ da coorte RaDC / IMRT vs. $27 \%$ na coorte IMRT recebendo odontologia convencional Cuidado).
Após tratamento dentário menor invasivo, não superior a grade Osteorradionecrose ou ressecções de mandíbula relacionadas com Osteorradionecrose ocorreram em nosso IMRT coorte de pacientes, como antes. O guia padrão mais invasivo linhas para tratamento dentário antes da radioterapia que têm sido válidos até agora não parece mais justificável para IMRT pacientes. Com base nos dados apresentados aqui, uma adaptação ao risco, menos abordagens invasivas para atendimento odontológico pré-IMRT é recomendada.

*O nível de evidência dos 4 artigos selecionados na amostra, é de 1B de acordo com o Nível de Evidência Científica por Tipo de Estudo - “Oxford Centre for Evidence-based Medicine". 


\section{DISCUSSÃO}

A radioterapia é um componente indispensável no manejo terapêutico dos cânceres de cabeça e pescoço. Dentre os efeitos adversos, destaca-se a ORN por sua prevalência (de 5 a 15\%), sua progressão e patogênese desconhecida. Sendo assim, a literatura aborda meios preventivos da ORN, dentre eles, a extração dentária antes do tratamento radioterápico. No entanto, há controvérsias diante de sua efetividade. Ao longo dos anos, acreditava-se que pacientes edêntulos tinham menor probabilidade em desenvolver a ORN, por remover possíveis focos de infecção (Batstone et al., 2011). Embora estudos como Nabil et al. (2011) evidenciam a redução de ORN em pacientes edêntulos, e sobretudo, usuários do tratamento com Oxigenoterapia Hiperbárica, estudos como Gallegos-Hernández et al. (2016), demonstraram que a ORN é uma doença multifatorial, e exodontias não são eficazes, devendo assim, ser evitadas.

Durante anos, a utilização de Plasma Rico em Plaquetas (PRP) era justificada como alternativa para o tratamento da ORN, visto que, seus fatores de crescimento eram benéficos para regeneração, além da presença de produto sanguíneo autólogo, no qual removeria o risco de infecções. Contudo, Batstone et al. (2011) ao analisarem o uso das PRP's concluíram que não são eficazes na prevenção da ORN, e tampouco, possuem benefício na cicatrização de feridas após extrações dentárias advindas do tratamento radioterápico. Ademais, Palma et al. (2020) evidenciaram ao analisar Fibrina rica em leucócitos, que o seu uso não fornece quaisquer benefícios, sendo recomendado o tratamento cirúrgico de remoção da lesão aliado ao medicamentoso.

Os dados alcançados no estudo de Delanian et al. (2011) indicaram satisfação no uso da associação de três drogas: Pentoxifilina, Tocoferol e Clodronato, conhecidas como PENTOCLO no tratamento da osteorradionecrose. Dessa forma, tal terapêutica apresentou eficácia considerável no tratamento de pacientes com lesões ósseas. Durante a pesquisa, o tratamento com esses medicamentos não foi suspenso devido a efeitos adversos. Além disso, houve uma regressão da lesão óssea exposta durante o tratamento, demonstrando eficiência ao longo de vários meses, levando até a cura e melhoria completa da mucosa. Ademais, após o rompimento do PENTOCLO, não foi observado nenhum efeito rebote. 
Nesse cenário, Pinto (2017) e Miniello (2016) encontraram efeitos similares, indicando que a união do clodronato, pentoxifilina e tocoferol reduziu significativamente a ORN, assim como observado anteriormente por Delanian et al. (2011). Assim, torna-se evidente que a pentoxifilina e o tocoferol são capazes de impedir o processo fibrótico juntamente com a diminuição no prejuízo na radiação, enquanto, o clorodonato é responsável por reduzir a reabsorção óssea e induzir a cicatrização óssea. Dessa forma, conclui-se que para o início do tratamento é realizada uma primeira fase com a administração de $2 \mathrm{~g}$ de amoxicilina associada com ácido clavulânico, $1 \mathrm{~g}$ de ciprofloxacina, $20 \mathrm{mg}$ de prednisolona e omeprazol, e $50 \mathrm{mg}$ de fluconazol. Após isso, é feita uma segunda fase onde se administra $800 \mathrm{~g}$ de pentoxifilina, 1000 UI de tocoferol, mais $1600 \mathrm{mg}$ de clodronato. Esse processo é feito cinco dias por semana e aos finais de semana é feita a administração da prednisolona 20 mg.

Houve o surgimento de uma técnica de radioterapia de intensidade modulada (IMRT), através dela é possível incidir altas doses de radiação determinados volumealvos. Isso é realizado, por meio de programação computadorizada e o uso de reconstruções tomográficas 3D. Teoricamente, com essa técnica haveria redução de efeitos adversos nos tecidos envolventes. No estudo de STUDER et al. (2011) observouse que posteriormente a um tratamento dentário menos agressivo, não foram encontrados graus superiores de ORN ou ressecções de mandíbulas em pacientes submetidos a IMRT. Nesse estudo, esperava-se obter um nível de incidência de ORN similar nos dois grupos estudados, já que esse tipo de efeito colateral surge em áreas expostas a altas doses de radiação. O número de dentes extraídos em cada grupo foi reduzido. Além disso, não houve quaisquer modificações ou adiamento no plano de radiação da tomografia computadorizada. Entretanto, nesse âmbito, de acordo com Owosho et al (2017) mesmo diante da técnica de radioterapia de intensidade modulada, doses acima de $60 \mathrm{~Gy}$, ou seja, altas doses de radiação continuam estatisticamente relacionadas com o aumento no surgimento de ORN. 


\section{CONCLUSÃO}

O tratamento da ORN vai variar de acordo com a extensão da lesão, gravidade e sintomatologia. Sendo assim, através desta revisão, constatou-se que: A fibrina rica em leucócitos e plaquetas (L-PRF) não é uma terapia efetiva para prevenção da Osteorradionecrose. Além disso, a associação de pentoxifilina, tocoferol e clodronato (PENTOCLO) é satisfatória e segura, como medicação para o tratamento da Osteorradionecrose.

Com isso, é possível afirmar a necessidade da realização de mais pesquisas acerca da temática, a fim de auxiliar na perpetuação do conhecimento dos profissionais de saúde, consequentemente, de forma a promover mudanças significativas e avanços no tratamento e manejo desses pacientes.

\section{REFERÊNCIAS}

BATSTONE, M.D et al. Platelet Rich Plasma for the Prevention of Osteoradionecrosis. A Double Blinded Randomized Cross over Controlled Trial. International Journal of Oral and Maxillofacial Surgery, Estados Unidos, ano 1, v. 41, p. 2-4, 1 jan. 2012.

BENNETT, M.H et al. Hyperbaric oxygen therapy for late radiation tissue injury. Cochrane Database Syst Rev., Australia, v. 1, 2017.

COOK, D. J. et al. Clinical recommendations using levels of evidence for antithrombotic agents. Chest, v. 108, p. 227-230, 1995

EL-RABBANY, M; DUCHNAY, M; RAZIEE, H.R; ZYCH, M. Interventions for preventing osteoradionecrosis of the jaws in adults receiving head and neck radiotherapy. Cochrane Database Syst Rev., USA, p. 1-6, 20 nov. 2021.

GALLEGOS-HERNÁNDEZ, J.F et al. [Mandibular osteoradionecrosis (ORN) as a side effect of head and neck cancer treatment: Factors that induce it]. Gaceta Medica De Mexico, Mexico, v. 152, p. 730-733, 6 dez. 2016.

KOLOKYTHAS, A; RASMUSSEN, J.T; REARDON, J; FENG, C. Management of osteoradionecrosis of the jaws with pentoxifylline-tocopherol: a systematic review of the literature and meta-analysis. . International Journal of Oral and Maxillofacial Surgery, [S. l.], ano 2, v. 48, p. 173-180, 2 fev. 2019. 
MINIELLO, T.G. Fatores Preditivos, prevalência e tratamento de osteorradionecrose em pacientes irradiados em região de cabeça e pescoço. Orientador: Fabio de Abreu Alves. 2016. 79 p. Dissertação (Mestrado) (Mestrado em Ciências) - Faculdade de Odontologia da Universidade de São Paulo, São Paulo, 2016.

MOHER, D. et al. Preferred reporting items for systematic reviews and meta-analyses: The PRISMA statement. PLoS Medicine, v. 6, n. 7, p. 1-6, 2009

OWOSHO, A.A et al. The Prevalence and Risk Factors Associated with Osteoradionecrosis of the Jaw in Oral and Oropharyngeal Cancer Patients Treated with Intensity-Modulated Radiation Therapy (IMRT): The Memorial Sloan Kettering Cancer Center Experience". Oral Oncology, [s. l.], v. 64, p. 44-51, 2017.

PALMA, L.F; MARCUCCI, M.; REMONDES, C.M; CHAMBRONE, L. Leukocyteand platelet-rich fibrin does not provide any additional benefit for tooth extraction in head and neck cancer patients post-radiotherapy: a randomized clinical trial. Medicina Oral, Patologia Oral Y Cirugia Bucal, Espanha, v. 25, n. 6, p. 799-804, 6 nov. 2020.

PINTO, R.F.S. ABORDAGENS TERAPÊUTICAS NA OSTEORRADIONECROSE DOS MAXILARES. Orientador: Pedro de Sousa Gomes. 2017. 52 p. Dissertação (Mestrado) (Mestrado Integrado em Medicina Dentária) - Faculdade de Medicina Dentária da Universidade do Porto, Porto, 2017.

ROBARD, L et al. Medical Treatment of Osteoradionecrosis of the Mandible by PENTOCLO: Preliminary Results. European Annals of Otorhinolaryngology, Head and Neck Diseases, [S. l.], v. 131, n. 6, p. 333-338, 1 dez. 2014.

SILVA, M.R.F. ESTUDO PROSPECTIVO RANDOMIZADO E DUPLO CEGO DE DOIS PROTOCOLOS DE COBERTURA ANTIBIÓTICA NA PREVENÇÃO DE OSTEORRADIONECROSE EM PACIENTES TRATADOS COM RADIOTERAPIA DE CABEÇA E PESCOÇO. Orientador: Prof. Dr. Fábio Abreu Alves. 2017. 71 p. Tese de Doutorado (Doutorado em Ciências) - Universidade Federal do Ceará, São Paulo, 2017.

STUDER, G; GLANZMANN, C; STUDER, S.P; GRÄTZ, KW; BREDELL, M; LOCHER, M.C; LÜTOLF, U.M; ZWAHLEN, R.A. Risk-adapted dental care prior to intensity-modulated radiotherapy (IMRT). Schweizer Monatsschrift Fur Zahnmedizin = Revue Mensuelle Suisse D'odonto-Stomatologie = Rivista Mensile Svizzera Di Odontologia E Stomatologia, [S. l.], ano 3, v. 121, p. 216-229, 1 jan. 2011. 
SYLVIE DELANIAN , M.D et al. COMPLETE RESTORATION OF REFRACTORY MANDIBULAR OSTEORADIONECROSIS BY PROLONGED TREATMENT WITH A PENTOXIFYLLINE-TOCOPHEROL-CLODRONATE COMBINATION (PENTOCLO): A PHASE II TRIAL. International Journal of Radiation Oncology, Biology, Physics, Estados Unidos, ano 3, v. 80, p. 832-839, 6 jul. 2011.

Recebido em: 15/112021

Aprovado em: 10/12/2021

Publicado em: 15/12/2021 